\title{
DIAGONALS OF SELF-ADJOINT OPERATORS
}

\author{
WILLIAM ARVESON* AND RICHARD V. KADISON
}

\begin{abstract}
The eigenvalues of a self-adjoint $n \times n$ matrix $A$ can be put into a decreasing sequence $\lambda=\left(\lambda_{1}, \ldots, \lambda_{n}\right)$, with repetitions according to multiplicity, and the diagonal of $A$ is a point of $\mathbb{R}^{n}$ that bears some relation to $\lambda$. The Schur-Horn theorem characterizes that relation in terms of a system of linear inequalities.

We give a new proof of the latter result for positive trace-class operators on infinite dimensional Hilbert spaces, generalizing results of one of us on the diagonals of projections. We also establish an appropriate counterpart of the Schur inequalities that relate spectral properties of self-adjoint operators in $I I_{1}$ factors to their images under a conditional expectation onto a maximal abelian subalgebra.
\end{abstract}

\section{Preface}

These are research notes that are not intended for publication in their present form. They summarize some of the results of a project begun by the authors that is directed toward finding an appropriate common generalization of the Schur-Horn theorem (for matrices) to operators on an infinite-dimensional Hilbert space, and to operators in finite factors, in a form that would generalize work of one of us on projections in $I I_{1}$ factors Kad02a, Kad02b. The results below were obtained in the fall of 2002. Other aspects of the project were presented in Section 5 of $\operatorname{Kad04}$.

This work is continuing, and remains unfinished. The following results are satisfactory in the case of type $I$ factors, but are incomplete for finite factors. We are making these partial results public since there is renewed interest in these directions She05] and it seems desirable to avoid duplication of effort. We also point out that the results of Section 4 overlap significantly with work of Andreas Neumann; and we thank Daniel Markiewicz for calling our attention to the paper Neu99 (also see Neu02]). The methods we use here differ in significant ways from the methods of [Neu99].

\section{The Results of Schur And Horn}

We begin by stating the key assertion of Theorem 5 from Alfred Horn's 1954 paper Hor54, which can be formulated as follows.

1991 Mathematics Subject Classification. 46L55, 46L09.

*supported by NSF grant DMS-0100487. 
Theorem 2.1 (Horn). Let $\lambda_{1} \geq \cdots \geq \lambda_{n}$ and $p_{1} \geq \cdots \geq p_{n}$ be two decreasing sequences of real numbers satisfying $p_{1}+\cdots+p_{k} \leq \lambda_{1}+\cdots+\lambda_{k}$, $1 \leq k \leq n-1$, and $p_{1}+\cdots+p_{n}=\lambda_{1}+\cdots+\lambda_{n}$. Then there is a selfadjoint $n \times n$ matrix with eigenvalues $\lambda_{1}, \ldots, \lambda_{n}$ whose diagonal entries are $p_{1}, \ldots, p_{n}$.

Another proof is offered at the end of this section. In a more coordinatefree formulation, Horn's theorem makes the following assertion. Let $A$ be a self-adjoint operator on an $n$-dimensional Hilbert space $H$ with eigenvalues $\lambda_{1} \geq \cdots \geq \lambda_{n}$, and let $p_{1} \geq \cdots \geq p_{n}$ be a decreasing sequence that relates to $\lambda$ as in the hypothesis of Theorem 2.1. Then there is an orthonormal basis $e_{1}, \ldots, e_{n}$ for $H$ such that

$$
\left\langle A e_{k}, e_{k}\right\rangle=p_{k}, \quad k=1, \ldots, n .
$$

The converse of Theorem 2.1 is also true, and this is the part of the composite Schur-Horn theorem that is attributed to Schur Sch23: If there is a self-adjoint $n \times n$ matrix $A$ with eigenvalue sequence $\lambda=\left(\lambda_{1}, \ldots, \lambda_{n}\right)$ with diagonal $p=\left(p_{1}, \ldots, p_{n}\right)$, both written in decreasing order, then the inequalities

$$
p_{1}+\cdots+p_{k} \leq \lambda_{1}+\cdots+\lambda_{k}, \quad 1 \leq k \leq n
$$

of the hypothesis of Theorem [2.1] are satisfied, with equality holding for $k=n$. That implication follows from classical estimates going back to Weyl Wey12 (see the proof of Theorem 4.1 below).

There are other formulations of the Schur-Horn theorem that borrow from classical inequalities Har34, the most notable one being the following. Given a sequence $\lambda=\left(\lambda_{1}, \ldots, \lambda_{n}\right)$ of real numbers, let $\mathcal{O}_{\lambda}$ be the set of all $n \times n$ self-adjoint matrices having eigenvalue sequence $\lambda$. Then the set $E\left(\mathcal{O}_{\lambda}\right)$ of all diagonals of matrices in $\mathcal{O}_{\lambda}$ is the convex hull $\Lambda$ of the set of points $\lambda \circ \pi \in \mathbb{R}^{n}$ obtained by permuting the components of $\lambda$. Schur's part of the Schur-Horn theorem becomes the assertion $E\left(\mathcal{O}_{\lambda}\right) \subseteq \Lambda$ while Theorem 2.1 implies $E\left(\mathcal{O}_{\lambda}\right) \supseteq \Lambda$. These formulations are discussed in [Hor54].

The Schur-Horn theorem has led to generalizations in several directions. In 1970, Kostant put it into the context of actions of compact Lie groups (which generalize the unitary group $U(n)$ ). Later Atiyah Ati82, and independently Guillemin and Sternberg [GS82, reformulated Kostant's result in the broader context of symplectic manifolds $M$ acted on by a torus $T$, and showed that for every moment map $\Phi$ for the $T$-action, the range of $\Phi$ is the convex hull of the images of the $T$-fixed points of $M$. See Knu00 for more detail.

There is some connection between the finite dimensional Schur-Horn theorem Hor54 and Horn's subsequent work on the eigenvalues of sums of matrices that culminated in the inequalities conjectured in Hor62, as described in Knu00. The Horn conjecture was recently proved, following work of Klyachko Kly98 and the proof of the saturation conjecture by Knutson and Tao KT99. Thus, it may be appropriate to point out that Friedland 
has obtained a generalization of Klyachko's results to finite sums of positive trace-class operators acting on infinite dimensional Hilbert spaces [Fri00.

The purpose of this paper is to discuss two infinite dimensional formulations of the Schur-Horn Theorem. In Sections 34 we present a generalization of the Schur-Horn theorem to positive trace class operators on infinite dimensional Hilbert spaces. In Sections 517, we reformulate these issues in the context of finite factors, and we establish appropriate versions of the Schur inequalities. The $I I_{1}$ version of Horn's result (Theorem 2.1) is left as an open problem.

The first author wishes to thank Allen Knutson for helpful comments about the Schur-Horn theorem including the sketch of a "calculus" proof, and for providing some key references.

Proof of Theorem 2.1. We show how one can deduce Theorem 2.1 from two results of $\operatorname{Kad02\mathrm {a}}$. Let $p=\left(p_{1}, \ldots, p_{n}\right)$ and $\lambda=\left(\lambda_{1}, \ldots, \lambda_{n}\right)$ be two decreasing sequences satisfying the hypotheses of Theorem [2.1. By lemma 5 of $\operatorname{Kad02a}$, there is a sequence of points $x_{1}, \ldots, x_{n}$ in $\mathbb{R}^{n}$ such that $x_{1}=\lambda$, $x_{n}=p$, and for $k=1, \ldots, n-1, x_{k+1}$ can be expressed in terms of $x_{k}$ as follows

$$
x_{k+1}=t_{k} \cdot x_{k}+\left(1-t_{k}\right) \cdot x_{k} \circ \tau_{k}
$$

where $t_{k}$ is a number in the unit interval, $\tau_{k}$ is a transposition in $S_{n}$, and where $x \circ \tau$ denotes $\left(x_{\tau(1)}, \ldots, x_{\tau(n)}\right) \in \mathbb{R}^{n}$.

Given $x_{1}=\lambda, \ldots, x_{n}=p \in \mathbb{R}^{n}, t_{1}, \ldots, t_{n-1} \in[0,1]$ and $\tau_{1}, \ldots, \tau_{n-1} \in S_{n}$ such that the relations (2.2) are satisfied, we exhibit a sequence of selfadjoint matrices $A_{1}, \ldots, A_{n}$ such that $A_{k}$ has eigenvalue list $\lambda$ and diagonal sequence $x_{k}$ as follows. Theorem 6 of Kad02a asserts the following: Given a self-adjoint $n \times n$ matrix $A=\left(a_{i j}\right)$ with diagonal sequence $x$, and given a transposition $\tau$ in $S_{n}$ and a number $t \in[0,1]$, then there is a unitary matrix $U$ such that the diagonal of $U A U^{*}$ is $t \cdot x+(1-t) \cdot x \circ \tau$. The proof exhibits $U=\left(u_{i j}\right)$ explicitly; if $\tau$ is the transposition $(i j)$ then $U$ coincides with the identity matrix except for the four terms $u_{i i}, u_{i j}, u_{j i}, u_{j j}$ specified by

$$
\left(\begin{array}{ll}
u_{i i} & u_{i j} \\
u_{j i} & u_{j j}
\end{array}\right)=\left(\begin{array}{cc}
z \cos \theta & \sin \theta \\
-z \sin \theta & \cos \theta
\end{array}\right)
$$

where $z$ is a complex number of absolute value 1 such that $z a_{i j}$ is pure imaginary, and where $\theta$ satisfies $\cos ^{2} \theta=t$. Let $A_{1}$ be the diagonal matrix with diagonal $\lambda=\left(\lambda_{1}, \ldots, \lambda_{n}\right)=x_{1}$. Given that $A_{1}, \cdots, A_{k}$ have been defined and satisfy the asserted conditions for $1 \leq k<n$, the above result implies that there is a unitary matrix $U_{k}$ such that

$$
\operatorname{diag}\left(U_{k} A_{k} U_{k}^{*}\right)=t_{k} \cdot x_{k}+\left(1-t_{k}\right) \cdot x_{k} \circ \tau_{k} .
$$

Setting $A_{k+1}=U_{k} A_{k} U_{k}^{*}$ and continuing inductively, we obtain a sequence of matrices $A_{1}, \ldots, A_{n}$ whose last term $A_{n}=U_{n-1} \cdots U_{1} A_{1} U_{1}^{*} \cdots U_{n-1}^{*}$ is a self-adjoint matrix having eigenvalue list $\lambda$ and diagonal $p$. 


\section{Part 1. Type $I_{\infty}$ factors}

We first give a generalization of the Schur-Horn theorem to the case of positive trace-class operators acting on a separable infinite-dimensional Hilbert space.

\section{3. $\mathcal{L}^{1}$-CLOSED UNITARY ORBITS}

Let $H$ be a separable Hilbert space and let $A$ be a positive compact operator on $H$. The sequence of eigenvalues of $A$ can be put into decreasing order, with repetitions according to the multiplicity of positive terms in the sequence, to obtain a sequence $\lambda=\left(\lambda_{1}, \lambda_{2}, \ldots\right)$ satisfying $\lambda_{1} \geq \lambda_{2} \geq \cdots \geq 0$, and we have

$$
\lambda_{1}+\lambda_{2}+\cdots=\operatorname{trace} A \in[0,+\infty] .
$$

Such a decreasing sequence $\lambda$ will be called an eigenvalue list. The preceding formula shows that $A$ is trace-class iff its eigenvalue list belongs to $\ell^{1}$, and the set of all eigenvalue lists in $\ell^{1}$ is a weak ${ }^{*}$-closed cone, the weak ${ }^{*}$-topology on $\ell^{1}$ arising from the identification of $\ell^{1}$ with the dual of $c_{0}$.

The eigenvalue list of $A$ fails to be a complete invariant for unitary equivalence because it fails to detect zero eigenvalues except when $A$ is of finite rank. For example, if $A$ has infinitely many positive terms $\lambda_{k}$ in its spectrum and has trivial kernel, then $A$ and $A \oplus \mathbf{0}$ (0 being an the zero operator on some space of positive dimension) cannot be unitarily equivalent despite the fact that they have the same eigenvalue list.

The state of affairs for trace-class operators is described as follows. We write $\mathcal{L}^{1}=\mathcal{L}^{1}(H)$ for the Banach space of all trace-class operators on a Hilbert space $H$ with respect to the trace norm

$$
\|A\|_{1}=\operatorname{trace}|A|
$$

$|A|$ denoting the positive square root of $A^{*} A$. Given an eigenvalue list $\lambda \in \ell^{1}$, $\mathcal{O}_{\lambda}$ will denote the set of all positive trace-class operators on $H$ having $\lambda$ as their eigenvalue list. Given a positive trace-class operator $A \in \mathcal{B}(H), \mathcal{O}(A)$ will denote the trace-norm closure of the unitary orbit of $A$

$$
\mathcal{O}(A)=\left\{U A U^{*}: U \in \mathcal{U}(H)\right\}^{-\|\cdot\|_{1}} .
$$

Two trace-class operators $A, B$ are said to be $\mathcal{L}^{1}$ - equivalent if there is a sequence of unitary operators $U_{1}, U_{2}, \ldots$ such that that $\left\|A-U_{n} B U_{n}^{*}\right\|_{1} \rightarrow 0$ as $n \rightarrow \infty$; equivalently, $\mathcal{O}(A)=\mathcal{O}(B)$.

Proposition 3.1. Let $A$ be a positive trace-class operator in $\mathcal{B}(H)$ and let $\lambda$ be the eigenvalue list of $A$.

(i) $\mathcal{O}(A)$ is a Polish topological space on which the unitary group of $H$ acts minimally.

(ii) $\mathcal{O}(A)=\mathcal{O}_{\lambda}$; in particular, the eigenvalue list is a complete invariant for $\mathcal{L}^{1}$-equivalence. 
(iii) Two positive trace-class operators $A, B$ are $\mathcal{L}^{1}$-equivalent iff $A \oplus \mathbf{0}$ and $B \oplus \mathbf{0}$ are unitarily equivalent, where $\mathbf{0}$ denotes the zero operator on an infinite dimensional Hilbert space.

(iv) If $\lambda$ has only finitely many nonzero terms, then $\mathcal{O}_{\lambda}$ consists of a single unitary orbit $\left\{U A U^{*}: U \in \mathcal{U}(H)\right\}$.

Proof. (i): $\mathcal{O}_{\lambda}$ is a closed subset of $\mathcal{L}^{1}$ and therefore a separable complete metric space. The fact that the orbit of every point of $\mathcal{O}(A)$ under the action of $\mathcal{U}(H)$ is dense in $\mathcal{O}(A)$ follows from the fact that $\mathcal{L}^{1}$-equivalence is an equivalence relation.

(ii): Let $B$ be another positive trace-class operator with eigenvalue list $\mu$. We have to show that $A$ and $B$ are $\mathcal{L}^{1}$-equivalent $\Longleftrightarrow \lambda=\mu$. For the implication $\Longrightarrow$ we make use of the semiclassical inequality

$$
\sum_{k=1}^{\infty}\left|\lambda_{n}-\mu_{n}\right| \leq\|A-B\|_{1},
$$

a proof of which can be found in the appendix of [Pow67]. Since $B$ can be closely approximated in the norm of $\mathcal{L}^{1}$ by operators unitarily equivalent to $A$, this inequality implies that $B$ must have the same eigenvalue list as $A$, hence $\mu=\lambda$. Conversely, if $A$ and $B$ are two positive trace-class operators with the same eigenvalue list $\lambda=\left(\lambda_{1}, \lambda_{2}, \ldots\right)$, then by the spectral theorem we can decompose $A$ and $B$ as follows

$$
A=A_{n}+R_{n}, \quad B=B_{n}+S_{n}
$$

where $A_{n}$ and $B_{n}$ are finite rank positive operators with eigenvalue list $\left(\lambda_{1}, \ldots, \lambda_{n}, 0,0, \ldots\right)$ and where the remainders $R_{n}$ and $S_{n}$ satisfy

$$
\left\|R_{n}\right\|_{1}=\left\|S_{n}\right\|_{1}=\sum_{k=n+1}^{\infty}\left|\lambda_{k}\right| .
$$

Since $A_{n}$ and $B_{n}$ are obviously unitarily equivalent for every $n=1,2, \ldots$ and since $\left\|R_{n}\right\|_{1}$ and $\left\|S_{n}\right\|_{1}$ tend to zero as $n \rightarrow \infty$, it follows that there is a sequence of unitary operators $U_{1}, U_{2}, \ldots$ such that $\left\|B-U_{n} A U_{n}^{*}\right\|_{1} \rightarrow 0$.

(iii) is a consequence of (ii), which asserts that $A$ and $B$ are $\mathcal{L}^{1}$-equivalent iff they have the same eigenvalue list. Indeed, it is obvious that if $A$ and $B$ have the same eigenvalue list $\lambda$ then $A \oplus \mathbf{0}$ and $B \oplus \mathbf{0}$ are unitarily equivalent; conversely, if $A \oplus \mathbf{0}$ and $B \oplus \mathbf{0}$ are unitarily equivalent then $A \oplus \mathbf{0}$ and $B \oplus \mathbf{0}$ must have the same eigenvalue list, hence so do $A$ and $B$.

Finally, note that (iii) $\Longrightarrow$ (iv), since if $A$ is a finite rank positive operator with eigenvalue list $\lambda$, then all but a finite number of components of $\lambda$ are zero, hence $A$ is unitarily equivalent to $A \oplus \mathbf{0}$, so that all operators in $\mathcal{O}_{\lambda}$ are unitarily equivalent. 


\section{Diagonals of Trace Class Operators}

Let $H$ be a separable Hilbert space and let $e_{1}, e_{2}, \ldots$ be an orthonormal basis for $H$. The sequence of projections $E_{k}=\left[e_{k}\right], k=1,2, \ldots$ generates a discrete maximal abelian subalgebra $\mathcal{A} \subseteq \mathcal{B}(H)$, and the map that replaces an operator $A$ with the diagonal part $\left(a_{11}, a_{22}, \ldots\right)$ of its matrix $a_{i j}=\left\langle A e_{j}, e_{i}\right\rangle, i, j=1,2, \ldots$ can be viewed as the unique trace preserving conditional expectation $E: \mathcal{B}(H) \rightarrow \mathcal{A}$ given by

$$
E(A)=\sum_{n=1}^{\infty} E_{n} A E_{n}
$$

The following result provides an operator-theoretic generalization of the Schur-Horn theorem. Another approach, not limited to trace-class operators, can be found in Neu99.

Theorem 4.1. Let $\mathcal{A}$ be a discrete maximal abelian von Neumann algebra in $\mathcal{B}(H)$, let $E: \mathcal{B}(H) \rightarrow \mathcal{A}$ be the trace-preserving conditional expectation on $\mathcal{A}$ and let $A \in \mathcal{B}(H)$ be a positive trace-class operator with eigenvalue list $\lambda=\left(\lambda_{1} \geq \lambda_{2} \geq \cdots\right)$. Then $E(\mathcal{O}(A))$ consists of all positive trace-class operators $B \in \mathcal{A}$ whose eigenvalue list $p=\left(p_{1} \geq p_{2} \geq \cdots\right)$ satisfies

$$
p_{1}+\cdots+p_{n} \leq \lambda_{1}+\cdots+\lambda_{n}, \quad n=1,2, \ldots
$$

together with

$$
p_{1}+p_{2}+\cdots=\lambda_{1}+\lambda_{2}+\cdots .
$$

We will deduce Theorem 4.1 from the following general assertion about diagonals of positive compact operators.

Theorem 4.2. Let $\mathcal{A} \subseteq \mathcal{B}(H)$ be a discrete maximal abelian algebra with natural conditional expectation $E: \mathcal{B}(H) \rightarrow \mathcal{A}$. Let $A \in \mathcal{B}(H)$ be a positive compact operator with eigenvalue list $\lambda=\left(\lambda_{1} \geq \lambda_{2} \geq \cdots\right)$, and let $B$ be a positive compact operator in $\mathcal{A}$. The following are equivalent.

(i) There is contraction $L \in \mathcal{B}(H)$ such that $E\left(L^{*} A L\right)=B$.

(ii) The eigenvalue list $p=\left(p_{1} \geq p_{2} \geq \cdots\right)$ of $B$ satisfies

$$
p_{1}+p_{2}+\cdots+p_{n} \leq \lambda_{1}+\lambda_{2}+\cdots+\lambda_{n}, \quad n=1,2, \ldots
$$

We require some eigenvalue estimates that go back to work of Weyl Wey12, Wey49. Let $A$ be a positive compact operator with eigenvalue list $\lambda_{1} \geq \lambda_{2} \geq \cdots$ and let $\mathcal{P}_{n}$ be the set of all $n$-dimensional projections in $\mathcal{B}(H)$. Then we have

$$
\sup _{P \in \mathcal{P}_{n}} \operatorname{trace} A P=\max _{P \in \mathcal{P}_{n}} \operatorname{trace} A P=\lambda_{1}+\cdots+\lambda_{n},
$$

the maximum being achieved on any $n$-dimensional projection whose range contains eigenvectors for $\lambda_{1}, \ldots, \lambda_{n}$. Ky Fan's version of this result can be found on p. 22 of Bha87.

The proof of Theorem 4.2 also requires a geometric result, asserting that if $p=\left(p_{1}, \ldots, p_{n}\right)$ and $\lambda=\left(\lambda_{1}, \ldots, \lambda_{n}\right)$ are two finite eigenvalue lists that 
satisfy the first $n$ inequalities (4.1), then the components of $\lambda$ can be reduced so as to preserve the first $n-1$ inequalities, with equality in the $n$ th.

Lemma 4.3. Let $p=\left(p_{1}, \ldots, p_{n}\right)$ and $\lambda=\left(\lambda_{1}, \ldots, \lambda_{n}\right)$ be two decreasing sequences of nonnegative reals of length $n=1,2, \ldots$ satisfying

$$
p_{1}+\cdots+p_{k} \leq \lambda_{1}+\cdots+\lambda_{k}, \quad k=1,2, \ldots, n .
$$

There is a decreasing sequence $\mu=\left(\mu_{1}, \ldots, \mu_{n}\right)$ such that

$$
0 \leq \mu_{k} \leq \lambda_{k}, \quad p_{1}+\cdots+p_{k} \leq \mu_{1}+\cdots+\mu_{k},
$$

for $1 \leq k \leq n$, and $p_{1}+\cdots+p_{n}=\mu_{1}+\cdots+\mu_{n}$.

Proof. We argue by induction, the case $n=1$ being obvious. Fix $n \geq 2$ and suppose that Lemma 4.3 is true for sequences of length $n-1$. Let $D$ be the set of all points $\mu=\left(\mu_{1}, \ldots, \mu_{n}\right) \in \mathbb{R}^{n}$ satisfying $\mu_{1} \geq \cdots \geq \mu_{n} \geq 0$ and $\mu_{k} \leq \lambda_{k}, 1 \leq k \leq n$, and consider the compact convex set $K \subseteq \mathbb{R}^{n}$

$$
K=\left\{\mu \in D: \mu_{1}+\cdots+\mu_{k} \geq p_{1}+\cdots+p_{k}, \quad k=1, \ldots, n-1\right\} .
$$

Since $f(x)=x_{1}+\cdots+x_{n}$ is a linear functional on $\mathbb{R}^{n}, f(K)$ is a closed interval $I \subseteq \mathbb{R}$. We have to show that $p_{1}+\cdots+p_{n} \in I$. For that, it suffices to show that there are points $x, y \in K$ such that $f(x) \leq p_{1}+\cdots+p_{n} \leq f(y)$. Setting $y=\lambda \in K$, we have $p_{1}+\cdots+p_{n} \leq \lambda_{1}+\cdots+\lambda_{n}=f(y)$ by (4.4). For $x$, use the induction hypothesis to obtain numbers $\mu_{1} \geq \cdots \geq \mu_{n-1} \geq 0$ satisfying $0 \leq \mu_{k} \leq \lambda_{k}, \mu_{1}+\cdots+\mu_{k} \geq p_{1}+\cdots+p_{k}, 1 \leq k \leq n-1$, and $\mu_{1}+\cdots+\mu_{n-1}=p_{1}+\cdots+p_{n-1}$. The point $x=\left(\mu_{1}, \ldots, \mu_{n-1}, 0\right)$ belongs to $K$ and satisfies $f(x)=p_{1}+\cdots+p_{n-1} \leq p_{1}+\cdots+p_{n}$.

Proof of Theorem 4.2. (i) $\Longrightarrow$ (ii): Let $e_{1}, e_{2}, \ldots$ be an orthonormal basis for $H$ with the property that $\left\langle B e_{j}, e_{j}\right\rangle=p_{k}, j=1,2, \ldots$ Fixing $k$ and letting $E$ be the projection onto the span of $e_{1}, \ldots, e_{k}$, we have

$p_{1}+\cdots+p_{k}=\operatorname{trace}(B E)=\operatorname{trace}\left(L^{*} A L E\right)=\operatorname{trace}\left(A L E L^{*}\right) \leq \operatorname{trace}(A F)$

where $F$ is the projection onto the range of the positive contraction $L E L^{*}$. Since $F$ is a projection of rank at most $k$, the estimate (4.3) implies

$$
\operatorname{trace}(A F) \leq \sup _{\operatorname{dim} F=k} \operatorname{trace}(A F)=\lambda_{1}+\cdots+\lambda_{k},
$$

and (ii) follows.

(ii) $\Longrightarrow$ (i): Let $B$ be a positive compact operator in $\mathcal{A}$ whose eigenvalue list $p=\left(p_{1} \geq p_{2} \geq \cdots\right)$ satisfies the inequalities (ii) and let $e_{1}, e_{2}, \ldots$ be an orthonormal basis for $H$ such that $\left[e_{1}\right],\left[e_{2}\right], \ldots$ are the minimal projections of $\mathcal{A}$. Since every permutation of the basis $\left\{e_{k}\right\}$ is implemented by a unitary operator $W \in \mathcal{B}(H)$ satisfying $W \mathcal{A} W^{*}=\mathcal{A}$, we may assume without essential loss that $B e_{k}=p_{k} e_{k}, k=1,2, \ldots$.

We construct a sequence of operators $L_{n} \in \mathcal{B}(H), n=1,2, \ldots$, as follows. Consider the spectral representation of $A$

$$
A=\sum_{k=1}^{\infty} \lambda_{k} \xi_{k} \otimes \bar{\xi}_{k}
$$


where $\xi_{1}, \xi_{2}, \ldots$ is an orthonormal sequence in $H$ consisting of eigenvectors of $A$. Fix $n$, let $H_{n}$ be the linear span of $\xi_{1}, \ldots, \xi_{n}$, and let $A_{n}$ be the restriction of $A$ to $H_{n}$. The eigenvalue list of $A_{n}$ is $\left(\lambda_{1}, \ldots, \lambda_{n}\right)$; so by Lemma 4.3. there is a decreasing sequence $\mu=\left(\mu_{1}, \ldots, \mu_{n}\right)$ satisfying $0 \leq \mu_{k} \leq \lambda_{k}$ for $1 \leq k \leq n$, and

$$
p_{1}+\cdots+p_{k} \leq \mu_{1}+\cdots+\mu_{k}, \quad k=1, \ldots, n,
$$

with equality holding for $k=n$. The sequence $\mu$ of course depends on $n$ but we suppress that in the notation since $\mu$ will soon disappear. Consider the operator $B_{n}$ defined on $H_{n}$ by requiring $B_{n} \xi_{k}=\mu_{k} \xi_{k}, 1 \leq k \leq n$. The eigenvalue list of $B_{n}$ dominates $\left(p_{1}, \ldots, p_{n}\right)$ as in the hypothesis of Horn's result Theorem 2.1. Thus there is an orthonormal basis $e_{1}^{(n)}, \ldots, e_{n}^{(n)}$ for $H_{n}$ such that

$$
\left\langle B_{n} e_{k}^{(n)}, e_{k}^{(n)}\right\rangle=p_{k}, \quad k=1, \ldots, n .
$$

Since $0 \leq B_{n} \leq A_{n}$ it follows that

$$
p_{k} \leq\left\langle A_{n} e_{k}^{(n)}, e_{k}^{(n)}\right\rangle=\left\langle A e_{k}^{(n)}, e_{k}^{(n)}\right\rangle, \quad k=1, \ldots, n .
$$

Let $L_{n} \in \mathcal{B}(H)$ be the operator defined by $L_{n} e_{k}=e_{k}^{(n)}$ for $k=1, \ldots, n$, and $L_{n}=0$ on the orthocomplement of $\left[e_{1}^{(n)}, \ldots, e_{n}^{(n)}\right]$.

We have constructed a sequence $L_{1}, L_{2}, \ldots$ of finite rank partial isometries in $\mathcal{B}(H)$ that satisfies the system of inequalities

$$
p_{k} \leq\left\langle A L_{n} e_{k}, L_{n} e_{k}\right\rangle, \quad n \geq k \geq 1 .
$$

Since the unit ball of $\mathcal{B}(H)$ is sequentially compact in its weak operator topology, there is a subsequence $n_{1}<n_{2}<\ldots$ and a contraction $L_{\infty} \in$ $\mathcal{B}(H)$ such that $\left\langle L_{n_{j}} \eta, \zeta\right\rangle \rightarrow\left\langle L_{\infty} \eta, \zeta\right\rangle$ as $j \rightarrow \infty$, for every $\eta, \zeta \in H$. We claim that $L_{\infty}$ satisfies

$$
p_{k} \leq\left\langle A L_{\infty} e_{k}, L_{\infty} e_{k}\right\rangle, \quad k=1,2, \ldots
$$

To see that, fix $k$ and note that for sufficiently large $j$, (4.6) implies

$$
p_{k} \leq\left\langle A L_{n_{j}} e_{k}, L_{n_{j}} e_{k}\right\rangle .
$$

As $j \rightarrow \infty, L_{n_{j}} e_{k}$ tends to $L_{\infty} e_{k}$ in the weak topology of $H$. Since $A$ is a compact operator, $\left\|A L_{n_{j}} e_{k}-A L_{\infty} e_{k}\right\| \rightarrow 0$ as $j \rightarrow \infty$; hence the inner products $\left\langle A L_{n_{j}} e_{k}, L_{n_{j}} e_{k}\right\rangle$ converge to $\left\langle A L_{\infty} e_{k}, L_{\infty} e_{k}\right\rangle$, and (4.7) follows.

Finally, choose $t_{1}, t_{2}, \cdots \in[0,1]$ such that $p_{k}=t_{k}\left\langle A L_{\infty} u_{k}, L_{\infty} u_{k}\right\rangle$ for every $k$. Letting $D \in \mathcal{B}(H)$ be the contraction defined by $D e_{k}=\sqrt{t_{k}} e_{k}$, $k \geq 1$, one finds that the operator $L=L_{\infty} D$ satisfies

$$
\left\langle L^{*} A L e_{k}, e_{k}\right\rangle=p_{k}, \quad k=1,2, \ldots,
$$

and the required formula

$$
E\left(L^{*} A L\right)=\sum_{k=1}^{\infty} E_{k} L^{*} A L E_{k}=\sum_{k=1}^{\infty} p_{k} E_{k}=B
$$

follows. 
Proof of Theorem 4.1. Let $E_{1}, E_{2}, \ldots$ be the minimal projections of $\mathcal{A}$ and let $e_{1}, e_{2}, \ldots$ be an orthonormal basis for $H$ such that $E_{k}$ is the projection $\left[e_{k}\right], k=1,2, \ldots$

We show first that for every positive trace class operator $A \in \mathcal{B}(H)$, the eigenvalue list $p=\left(p_{1}, p_{2}, \ldots\right)$ of $B=E(A)$ must satisfy (4.1) and (4.2). By permuting the elements of the basis $\left\{e_{k}\right\}$ appropriately and changing notation, we may assume that $B e_{k}=p_{k} e_{k}, k=1,2, \ldots$ Let $P_{n}$ be the projection on $\left[e_{1}, \ldots, e_{n}\right]$. Since $A$ is a positive compact operator with eigenvalue list $\lambda$, we can make use of (4.3) to write

$$
p_{1}+\cdots+p_{n}=\sum_{k=1}^{n}\left\langle B e_{k}, e_{k}\right\rangle=\operatorname{trace} A P_{n} \leq \lambda_{1}+\cdots+\lambda_{n} .
$$

Moreover, $p_{1}+p_{2}+\cdots=\left\langle A u_{1}, u_{1}\right\rangle+\left\langle A u_{2}, u_{2}\right\rangle+\cdots=\operatorname{trace} A=\lambda_{1}+\lambda_{2}+\cdots$.

Conversely, let $p$ and $\lambda$ be two summable eigenvalue lists that satisfy (4.1) - (4.2), and let $B$ be a positive trace-class operator in $\mathcal{A}$ with list $p$. Again, by relabeling the orthonormal basis $\left\{e_{k}\right\}$, we may assume that $B e_{k}=p_{k} e_{k}, k=1,2, \ldots$ Choose any positive trace-class operator $A \in$ $\mathcal{B}(H)$ having eigenvalue list $\lambda$, and let $P$ be the projection onto the closure of $A H$. Theorem 4.2 implies that there is a contraction $L \in \mathcal{B}(H)$ satisfying $p_{k}=\left\langle A L e_{k}, L e_{k}\right\rangle$ for $k \geq 1$. By replacing $L$ with $P L$ if necessary, we may also assume that $L H$ is contained in $P H$, and in that case we claim:

$$
L L^{*}=P .
$$

Indeed, $P-L L^{*} \geq 0$ because $L$ is a contraction whose range is contained in $P H$, and it suffices to show that the positive operator $A^{1 / 2}\left(P-L L^{*}\right) A^{1 / 2}=$ $A-A^{1 / 2} L L^{*} A^{1 / 2}$ has trace zero; equivalently, trace $A^{1 / 2} L L^{*} A^{1 / 2}=\operatorname{trace} A$. Using trace $X X^{*}=\operatorname{trace} X^{*} X$ for $X=A^{1 / 2} L$, we have

$$
\begin{aligned}
\operatorname{trace} A^{1 / 2} L L^{*} A^{1 / 2} & =\operatorname{trace} L^{*} A L=\sum_{n=1}^{\infty}\left\langle A L e_{n}, L e_{n}\right\rangle \\
& =p_{1}+p_{2}+\cdots=\lambda_{1}+\lambda_{2}+\cdots=\operatorname{trace} A .
\end{aligned}
$$

$L$ is a co-isometry by (4.8); hence it can be changed into a unitary operator $U: H \rightarrow H \oplus \operatorname{ker} L$ by making use of the projection $Q: H \rightarrow \operatorname{ker} L$ as follows: $U \xi=L \xi \oplus Q \xi, \xi \in H$. Now consider the operator

$$
A_{0} \oplus \mathbf{0} \in \mathcal{B}(P H \oplus \operatorname{ker} L),
$$

$A_{0}$ denoting the restriction of $A$ to $P H=\overline{A H}$. Since $U e_{k}=L e_{k} \oplus Q e_{k}$, $k=1,2, \ldots$, we have

$$
\left\langle\left(A_{0} \oplus \mathbf{0}\right) U e_{k}, U e_{k}\right\rangle=\left\langle A L e_{k}, L e_{k}\right\rangle=p_{k}, \quad k=1,2, \ldots
$$

Therefore $U^{*}\left(A_{0} \oplus \mathbf{0}\right) U$ is a positive trace class operator in $\mathcal{B}(H)$ satisfying

$$
E\left(U^{*}\left(A_{0} \oplus \mathbf{0}\right) U\right)=\sum_{k=1}^{\infty} p_{k} E_{k}=B .
$$


Since $U^{*}\left(A_{0} \oplus \mathbf{0}\right) U$ has the same eigenvalue list as $A$, Proposition 3.1 implies that it must belong to $\mathcal{O}(A)$, and the proof is complete.

In the series Kad02a, Kad02b, one of us carried out a study of the possible diagonals of projections acting on a separable Hilbert space. The results of this paper do not address the most difficult case where the projection has infinite rank and infinite co-rank; but Theorem 4.1 does give the result of Theorem 13 of $\mathrm{Kad02b}$, as follows. We formulate that in terms of the Hilbert space $\ell^{2}$ and its standard orthonormal basis $\left(u_{k}\right)_{k \geq 1}$, with the associated realization of operators as matrices relative to this basis.

Corollary 4.4. Let $p=\left(p_{1}, p_{2}, \ldots\right)$ be a sequence of numbers in the unit interval $0 \leq p_{k} \leq 1$, and let $m$ be a positive integer. The following are equivalent

(i) There is a rank $m$ projection $P \in \mathcal{B}\left(\ell^{2}\right)$ whose matrix has $p$ as its diagonal.

(ii) $p_{1}+p_{2}+\cdots=m$.

Proof. We prove the nontrivial implication (ii) $\Longrightarrow$ (i). Since (ii) implies that the sequence $p_{n}$ converges to zero and since permutations of $\mathbb{N}$ are implemented by unitary operators on $\ell^{2}(\mathbb{N})$ in the obvious way, it suffices to address the case where the sequence is decreasing $p_{1} \geq p_{2} \geq \cdots$. The eigenvalue list of a projection of rank $m$ is

$$
\lambda=(\underbrace{1, \ldots, 1}_{m \text { times }}, 0,0, \ldots),
$$

and $\mathcal{O}_{\lambda}$ consists of all rank $m$ projections in $\mathcal{B}\left(\ell^{2}\right)$. The hypothesis (ii), together with $0 \leq p_{k} \leq 1$, implies that $p_{1}+\cdots+p_{n} \leq \lambda_{1}+\cdots+\lambda_{n}$ holds for every $n \geq 1$. Hence Theorem 4.1 implies that there is an operator in $\mathcal{O}_{\lambda}$ with diagonal sequence $p$.

\section{Part 2. Type $I I_{1}$ Factors}

We turn now to the case of self-adjoint operators $A$ in a finite factor. In this context, the appropriate counterpart of the eigenvalue list is a finite positive measure on the real line, called the spectral distribution of $A$. After working out the basic properties of the spectral distribution and relating it to approximate unitary equivalence, we establish a generalization of the Schur inequalities for $I I_{1}$ factors.

\section{SpeCtral Distribution of A SELF-ADJOINT OPERATOR}

In the remainder of this paper we work within the context of a finite factor $\mathcal{M}$ with normalized trace $\tau$. For every self-adjoint operator $A \in \mathcal{M}$ there is a unique probability measure $m_{A}$ on the Borel subsets of the real line whose moments are given by

$$
\int_{-\infty}^{\infty} \lambda^{n} d m_{A}(\lambda)=\tau\left(A^{n}\right), \quad n=0,1,2, \ldots
$$


Definition 5.1. The measure $m_{A}$ is called the spectral distribution of $A$.

The purpose of these section is to discuss the basic properties of this invariant. The spectral distribution is the appropriate generalization to $I I_{1}$ factors of the eigenvalue list invariant of self-adjoint $n \times n$ matrices. Indeed, if $A$ is a self-adjoint $n \times n$ matrix with eigenvalue list $\Lambda=\left\{\lambda_{1} \geq \lambda_{2} \geq \cdots\right\}$, then $m_{A}$ is the discrete measure

$$
m_{A}=\frac{1}{n}\left(\delta_{\lambda_{1}}+\cdots+\delta_{\lambda_{n}}\right)
$$

$\delta_{\lambda}$ denoting the unit point mass concentrated at $\lambda \in \mathbb{R}$. Equivalently, $m_{A}$ assigns mass to singletons $\{\lambda\}$ of $\mathbb{R}$ as follows

$$
m_{A}(\lambda)= \begin{cases}\frac{1}{n}(\text { multiplicity of } \lambda), & \text { if } \lambda \in \sigma(A) \\ 0, & \text { otherwise. }\end{cases}
$$

We require the following observation, which asserts that the spectral distribution of an operator in a $I I_{1}$ factor can be arbitrary.

Proposition 5.2. Let $\mathcal{A} \subseteq \mathcal{M}$ be a MASA in a $I I_{1}$ factor and let $m$ be a compactly supported probability measure on the real line. Then there is a self-adjoint operator $A \in \mathcal{A}$ such that $m=m_{A}$.

Proof. $\mathcal{A}$ contains a countably-generated nonatomic subalgebra, which must be isomorphic to $L^{\infty}[0,1]$ in such a way that the restriction of the trace $\tau$ corresponds to the state of $L^{\infty}[0,1]$ given by

$$
\tau(f)=\int_{0}^{1} f(x) d x, \quad f \in L^{\infty}[0,1] .
$$

Thus it suffices to show that there is a real-valued function $f \in L^{\infty}[0,1]$ such that $\int_{0}^{1} f(x)^{n} d x=\int \lambda^{n} d m(\lambda)$ for $n=0,1, \ldots$ or equivalently, for every Borel set $S \subseteq \mathbb{R}$,

$$
\tau\{x \in[0,1]: f(x) \in S\}=m(S),
$$

where we abuse notation slightly by also writing $\tau$ for Lebesgue measure on the unit interval. Let $K$ be the closed support of $m$. The pair $(K, m)$ defines a separable measure algebra which may have a finite or countable number of atoms. On the other hand, $([0,1], \tau)$ gives rise to a nonatomic separable measure algebra. Letting $x_{1}, x_{2}, \ldots$ be the points of $K$ having positive $m$ measure, we find a sequence of disjoint Borel sets $E_{1}, E_{2}, \cdots \subseteq[0,1]$ such that $\tau\left(E_{k}\right)=m\left(\left\{x_{k}\right\}\right)$ for all $k$. Define $f$ on $\cup_{k} E_{k}$ so that it takes on the constant value $x_{k}$ throughout $E_{k} . f$ is a measure-preserving map of $\cup_{k} E_{k}$ onto the atomic part of $(K, \tau)$. Since $\tau\left([0,1] \backslash \cup_{k} E_{k}\right)=m\left(K \backslash\left\{x_{1}, x_{2}, \ldots\right\}\right)$ and since the remaining parts of both measure spaces are nonatomic and separable, there is a surjective Borel map of $[0,1] \backslash \cup_{n} E_{n}$ onto $K \backslash\left\{x_{1}, x_{2}, \ldots\right\}$ that pushes Lebesgue measure forward to $m$, and we can use this map to extend the definition of $f$ to all of $[0,1]$ in the obvious way. 
The eigenvalue list is a complete invariant for $\mathcal{L}^{1}$-equivalence of positive trace-class operators. We now show that the spectral distribution invariant occupies a similar position.

Definition 5.3. Operators $A, B \in \mathcal{M}$ are said to be approximately equivalent if there is a sequence of unitary operators $U_{1}, U_{2}, \ldots$ in $\mathcal{M}$ such that

$$
\lim _{n \rightarrow \infty}\left\|U_{n} A U_{n}^{*}-B\right\|=0 .
$$

The set of all operators in the norm-closed unitary orbit of $A$ is written $\mathcal{O}_{A}$.

Theorem 5.4. Let $A$ be a self-adjoint operator in $\mathcal{M}$, let $m_{A}$ be the spectral distribution of $A$, and let $\mathcal{O}_{A}$ be the norm-closed unitary orbit of $A$. Then $\mathcal{O}_{A}$ is closed in the strong operator topology, and consists of all self-adjoint operators $B \in \mathcal{M}$ satisfying $m_{B}=m_{A}$.

Before giving the proof we collect an elementary observation.

Lemma 5.5. Let $E_{1} \leq E_{2} \leq \cdots \leq E_{n}$ and $F_{1} \leq F_{2} \leq \cdots \leq F_{n}$ be two linearly ordered sets of projections in $\mathcal{M}$ such that $\tau\left(E_{k}\right)=\tau\left(F_{k}\right)$ for $k=$ $1, \ldots, n$. Then there is a unitary operator $U$ in $\mathcal{M}$ such that $U E_{k} U^{*}=F_{k}$, $k=1, \ldots, n$.

Proof. By adjoining the identity to the end of each list if necessary, we can assume that $E_{n}=F_{n}=1$. Setting $E_{0}=F_{0}=0$, the hypothesis implies that $\tau\left(E_{k}-E_{k-1}\right)=\tau\left(F_{k}-F_{k-1}\right)$ for each $k=1, \ldots, n$. Since $\mathcal{M}$ is a finite factor, projections with the same trace must be Murray-von Neumann equivalent. Thus there are partial isometries $U_{1}, \ldots, U_{n} \in \mathcal{M}$ with $U_{k}^{*} U_{k}=E_{k}-E_{k-1}$ and $U_{k} U_{k}^{*}=F_{k}-F_{k-1}$ for all $k$. The projections $U_{1}^{*} U_{1}, \ldots, U_{n}^{*} U_{n}$ add up to $E_{n}=\mathbf{1}$, and similarly $U_{1} U_{1}^{*}+\cdots+U_{n} U_{n}^{*}=\mathbf{1}$. It follows that $W=U_{1}+\cdots+U_{n}$ is a unitary operator in $\mathcal{M}$ with the property $W\left(E_{k}-E_{k-1}\right) W^{*}=F_{k}-F_{k-1}$ for every $k=1, \ldots, n$, hence $W E_{k} W^{*}=F_{k}$ for $k=1, \ldots, n$.

Proof of Theorem 5.4. We will show that a self-adjoint operator $B$ belongs to $\mathcal{O}_{A}$ iff $m_{B}=m_{A}$. Once that is established, it will follow that $\mathcal{O}_{A}$ is strongly closed, because the relation $m_{B}=m_{A}$ is characterized by the sequence of equations

$$
\tau\left(B^{n}\right)=\tau\left(A^{n}\right), \quad n=0,1,2, \ldots
$$

and each of the monomials $B \mapsto \tau\left(B^{n}\right)$ is strongly continuous on bounded subsets of $\mathcal{M}$.

Every operator $B \in \mathcal{M}$ that is unitarily equivalent to $A$ must satisfy the formulas (5.4), and hence a norm-limit of such operators will satisfy the same formulas. It follows that $m_{B}=m_{A}$ for every $B$ in the norm-closed unitary orbit of $A$.

Conversely, let $A, B$ be self-adjoint operators of $\mathcal{M}$ satisfying $m_{A}=m_{B}$, and let $[a, b]$ be an interval with the property that $m_{A}=m_{B}$ is supported 
in the interior $(a, b)$. Then the spectra of $A$ and $B$ are both contained in $(a, b)$. For every $a \leq t \leq b$ let $E_{t}$ (resp. $F_{t}$ ) be the spectral projection of $A$ (resp. $B$ ) corresponding to the subinterval $[a, t] \subseteq \mathbb{R}$. Then by hypothesis we have

$$
\tau\left(E_{t}\right)=m_{A}([a, t])=m_{B}([a, t])=\tau\left(F_{t}\right), \quad a \leq t \leq b .
$$

Given $\epsilon>0$ we can find a partition $a=t_{0}<t_{1}<\cdots<t_{n}=b$ of $[a, b]$ fine enough that

$$
\left|t-\sum_{k-1}^{n} t_{k} \chi_{\left(t_{k-1}, t_{k}\right]}(t)\right| \leq \epsilon, \quad a \leq t \leq b .
$$

Letting $A_{0}, B_{0}$ be the operators

$$
A_{0}=\sum_{k=1}^{n} t_{k}\left(E_{t_{k}}-E_{t_{k-1}}\right), \quad B_{0}=\sum_{k=1}^{n} t_{k}\left(F_{t_{k}}-F_{t_{k-1}}\right),
$$

we find that $\left\|A-A_{0}\right\| \leq \epsilon$ and $\left\|B-B_{0}\right\| \leq \epsilon$. (5.5) implies $\tau\left(E_{t_{k}}\right)=\tau\left(F_{t_{k}}\right)$ for every $k$, so by the Lemma there is a unitary operator $W \in \mathcal{M}$ such that $W E_{t_{k}} W^{*}=F_{t_{k}}$ for all $k$, hence $W A_{0} W^{*}=B_{0}$. An obvious estimate now implies $\left\|W A W^{*}-B\right\| \leq 2 \epsilon$, and since $\epsilon$ is arbitrary it follows that $A$ and $B$ are approximately equivalent.

Theorem 5.4 implies that the spectral distribution is a complete invariant for approximate unitary equivalence, and it is natural to ask if two self-adjoint operators that are approximately equivalent must be unitarily equivalent, or at least conjugate by way of a $*$-automorphism. The following class of examples shows that the answer is no.

Example. Let $\mathcal{R}$ be a $I I_{1}$ factor and let $\mathcal{A}$ and $\mathcal{B}$ be two MASAs in $\mathcal{R}$ that are not conjugate by way of an automorphism of $\mathcal{R}$. For example, $\mathcal{A}$ can be taken to be a regular MASA and $\mathcal{B}$ a singular one. Since both $\mathcal{A}$ and $\mathcal{B}$ are isomorphic to $L^{\infty}[0,1]$ by way of an isomorphism that carries the trace to Lebesgue measure, it follows that a) there is a $*$-isomorphism $\alpha$ of $\mathcal{A}$ onto $\mathcal{B}$ satisfying $\tau(\alpha(X))=\tau(X)$ for all $X \in \mathcal{A}$, and b) $\mathcal{A}$ is the von Neumann algebra $W^{*}(A)$ generated by a single self-adjoint operator $A$. Let $B=\alpha(A)$. Then $\tau\left(A^{n}\right)=\tau\left(B^{n}\right)$ for every $n=0,1,2, \ldots$ and hence $m_{A}=m_{B}$. It follows from Theorem 5.4 that $A$ and $B$ are approximately equivalent. On the other hand, there is no unitary operator $U \in \mathcal{R}$ satisfying $U A U^{*}=B$, since that would imply that $\theta(X)=U X U^{*}$ is an automorphism of $\mathcal{R}$ that carries $\mathcal{A}=W^{*}(A)$ onto $\mathcal{B}=W^{*}(B)$.

\section{SChUR-TyPe INEQUALITIES FOR $I I_{1}$ FACTORS}

The purpose of this section is to formulate an appropriate counterpart of the Schur inequalities for self-adjoint operators in $I I_{1}$ factors. This is not the only formulation possible, and we refer the reader to Section 5 of $\mathrm{Kad04}$ for an alternate approach. Here, we seek to formulate the Schur inequalities 
in terms of spectral distributions. The latter formulation is based on the following observations.

Proposition 6.1. For any two compactly supported probability measures $m$, $n$ on the real line, the following are equivalent:

(i) $m$ and $n$ have the same first moment

$$
\int_{\mathbb{R}} \lambda d m(\lambda)=\int_{\mathbb{R}} \lambda d n(\lambda)
$$

and for every $t \in \mathbb{R}$ we have

$$
\int_{t}^{\infty} m([s, \infty)) d s \leq \int_{t}^{\infty} n([s, \infty)) d s .
$$

(ii) $m$ and $n$ have the same first moment, and for every $t \in \mathbb{R}$ we have

$$
\int_{[t, \infty)}(\lambda-t) d m(\lambda) \leq \int_{[t, \infty)}(\lambda-t) d n(\lambda)
$$

(iii) For every continuous convex function defined on a closed interval $I=[c, d]$ that supports both measures $m$ and $n$, we have

$$
\int_{I} f(\lambda) d m(\lambda) \leq \int_{I} f(\lambda) d n(\lambda) .
$$

Proof. The equivalence (i) $\Longleftrightarrow$ (ii) is a consequence of the classic integration by parts formula of Riemann-Stieltjes integration, which can be applied as follows. Fix $t \in \mathbb{R}$, let $m$ be a compactly supported measure defined on $\mathbb{R}$, and choose $a, b \in \mathbb{R}$ so that $a<t<b$ and such that $(a, b)$ contains the closed support of both $m$ and $n$. Let $\alpha: \mathbb{R} \rightarrow \mathbb{R}$ be the decreasing function $\alpha(s)=m([s, \infty))$ and let $f$ be the continuous increasing function $f(s)=\max (s-t, 0)$. An application of Theorem 9-6 of Apo57 gives

$$
\int_{a}^{b} f(x) d \alpha(x)+\int_{a}^{b} \alpha(x) d f(x)=f(b) \alpha(b)-f(a) \alpha(a) .
$$

In this case, $\alpha(b)=f(a)=0$, and straightforward computations show that

$$
\begin{aligned}
& \int_{a}^{b} f(x) d \alpha(x)=-\int_{[t, \infty]}(x-t) d m(x), \\
& \int_{a}^{b} \alpha(x) d f(x)=\int_{t}^{\infty} m([x, \infty)) d x .
\end{aligned}
$$

It follows that

$$
\int_{[t, \infty]}(\lambda-t) d m(\lambda)=\int_{t}^{\infty} m([s, \infty)) d s,
$$

and the equivalence of (i) and (ii) follows.

The equivalence (ii) $\Longleftrightarrow$ (iii) follows from the basic fact that a continuous real-valued convex function $f$ defined on a compact interval $I \subseteq \mathbb{R}$ can be uniformly approximated on $I$ by functions

$$
f(\lambda)=a+b \lambda+g(\lambda)
$$


where $a$ and $b$ are real constants and $g$ belongs to the cone generated by the "angular" functions

$$
g_{t}(\lambda)=\max (\lambda-t, 0)=(\lambda-t) \chi_{[t, \infty)}(\lambda), \quad t \in \mathbb{R} .
$$

To see how the approximation (6.1) is achieved, one first approximates $f$ uniformly on $I$ with a twice continuously differentiable convex function $g$. Since $g^{\prime}$ is an increasing function, it can be uniformly approximated by an increasing step function having the form $a+h(\lambda)$ where $a$ is constant and $h$ belongs to the cone generated by the step functions $\chi_{[t, \infty)}, t \in \mathbb{R}$. After one integrates this approximation of $g^{\prime}$ one obtains an approximation to $g(\lambda)$ of the form $a \lambda+b+\int h(\lambda) d \lambda$. Moreover, since the indefinite integral of a step function $\chi_{[t, \infty)}(\lambda)$ has the form $c+g_{t}(\lambda)$ where $c$ is a constant, this approximation of $g$ has the form (6.1).

Definition 6.2. Let $m$ and $n$ be two compactly supported probability measures on the real line. We write $m \preceq n$ if $\int_{\mathbb{R}} \lambda d m(\lambda)=\int_{\mathbb{R}} \lambda d n(\lambda)$, and

$$
\int_{t}^{\infty} m([s, \infty)) d s \leq \int_{t}^{\infty} n([t, \infty)) d s, \quad t \in \mathbb{R} .
$$

The relation $\preceq$ is obviously a partial ordering on the set of all compactly supported probability measures on the real line. Given two self-adjoint operators $A, B$ in a $I I_{1}$ factor $\mathcal{M}$, we interpret the relation $m_{A} \preceq m_{B}$ as the appropriate counterpart of the Schur inequalities (2.1) that relate the eigenvalue lists of $A$ and $B$. This interpretation is justified by Proposition 6.1 and the following remarks.

Remark 6.3 (Relation to the classical inequalities of Schur). Let $\tau$ be the normalized trace on the matrix algebra $M_{n}(\mathbb{C})$, and let $A$ and $B$ be selfadjoint $n \times n$ matrices. We have discussed the relation between the eigenvalue list of $A$ and the spectral distribution $m_{A}$ in Section 5. We now examine the relation between the integrals

$$
\int_{t}^{\infty} m_{A}([s, \infty)) d s
$$

appearing in (6.2) and the eigenvalue list $\lambda_{1} \geq \cdots \geq \lambda_{n}$ of $A$. For simplicity, we consider the case where the eigenvalues are simple ones. For every $t$ in the interval $\lambda_{k+1}<t<\lambda_{k}$ one has $m([t, \infty))=k$, and after a straightforward integration and cancellation one finds that for $\lambda_{k-1}<t<\lambda_{k}$,

$$
\int_{t}^{\infty} m_{A}([s, \infty)) d s=\lambda_{1}+\lambda_{2}+\cdots+\lambda_{k}-k t .
$$

Let $B$ be another self-adjoint matrix with eigenvalue list $\mu_{1} \geq \cdots \geq \mu_{n}$. The preceding formula shows that the system of inequalities

$$
\int_{t}^{\infty} m_{A}([s, \infty)) d s \leq \int_{t}^{\infty} m_{B}([s, \infty)) d s, \quad t \in \mathbb{R}
$$


differs somewhat from the system of classical Schur inequalities

$$
\lambda_{1}+\cdots+\lambda_{k} \leq \mu_{1}+\cdots+\mu_{k}, \quad k=1,2, \ldots, n .
$$

However, if $A$ and $B$ have the same trace $\lambda_{1}+\cdots+\lambda_{n}=\mu_{1}+\cdots+\mu_{n}$, then the inequalities (6.3) are equivalent to the Schur inequalities (6.4) because they are equivalent to the inequalities of assertion (iii) of Proposition 6.1 That is a consequence of classical results of Hardy, Littlewood and Polya which are summarized in Theorem 1 of Hor54. The relevant result asserts that for two finite eigenvalue lists

$$
\left\{\lambda_{1} \geq \cdots \geq \lambda_{n}\right\}, \quad\left\{\mu_{1} \geq \cdots \geq \mu_{n}\right\}
$$

which satisfy $\lambda_{1}+\cdots+\lambda_{n}=\mu_{1}+\cdots+\mu_{n}$, the following are equivalent:

(1) $\lambda_{1}+\cdots+\lambda_{k} \leq \mu_{1}+\cdots+\mu_{k}$, for every $k=1, \ldots, n$.

(2) For every convex function $f$ defined on an interval containing all $\lambda_{i}$ and $\mu_{j}$, one has

$$
\sum_{k=1}^{n} f\left(\lambda_{k}\right) \leq \sum_{k=1}^{n} f\left(\mu_{k}\right) .
$$

Thus the system of inequalities (6.2) are appropriate generalizations of the Schur inequalities 6.4 to $I I_{1}$ factors.

\section{Proof of the Schur inequalities}

We require a convexity inequality for operators in a $I I_{1}$ factor. While related results can be found in the literature, we have been unable to find references appropriate for this particular result, and we include a proof for completeness. Let $\mathcal{A}$ be a maximal abelian self-adjoint subalgebra of a $I I_{1}$ factor $\mathcal{M}$ with normalized trace $\tau$, and let $E: \mathcal{M} \rightarrow \mathcal{A}$ be the $\tau$-preserving conditional expectation.

Proposition 7.1. Let $f$ be a real-valued continuous convex function defined on a compact interval $I=[a, b] \subseteq \mathbb{R}$. Then for every self-adjoint operator $A \in \mathcal{M}$ with spectrum contained in $I$, the spectrum of $E(A)$ is also contained in $I$ and we have

$$
f(E(A)) \leq E(f(A)) .
$$

Proof. For every self-adjoint operator $A \in \mathcal{M}$, we write $A_{+}$for the positive part of $A$, defined by $A_{+}=A P_{+}=P_{+} A$ where $P_{+}$is the spectral projection of $A$ associated with the nonnegative real axis $[0, \infty)$. We claim first that

$$
E(A)_{+} \leq E\left(A_{+}\right) .
$$

Indeed, in the natural ordering of self-adjoint operators in $\mathcal{M}$ we have $A \leq A_{+}$and hence $E(A) \leq E\left(A_{+}\right)$. Thus $E\left(A_{+}\right)$is a positive operator dominating $E(A)$. Since $\mathcal{A}$ is abelian, $E(A)_{+}$is the smallest positive operator in $\mathcal{A}$ that dominates $E(A)$, and (17.2) follows. 
In order to prove (7.1), choose $r \in \mathbb{R}$ and let $g_{r}(\lambda)=\max (\lambda-r, 0)$. We may apply (7.2) to the operator $A-r \mathbf{1}$ to obtain

$$
g_{r}(E(A))=(E(A)-r \mathbf{1})_{+}=E(A-r \mathbf{1})_{+} \leq E\left((A-r \mathbf{1})_{+}\right)=E\left(g_{r}(A)\right) .
$$

It follows that for every convex function $f_{0}: \mathbb{R} \rightarrow \mathbb{R}$ of the form

$$
f_{0}(\lambda)=a+b \lambda+\sum_{k=1}^{n} c_{k} g_{r_{k}}(\lambda)
$$

where $a, b, r_{1}, \ldots, r_{n} \in \mathbb{R}$ and $c_{1}, \ldots, c_{n} \geq 0$, one has

$$
f_{0}(E(A)) \leq E\left(f_{0}(A)\right) \text {. }
$$

Since every continuous convex function $f:[a, b] \rightarrow \mathbb{R}$ can be uniformly approximated by functions $f_{0}$ of the form (7.3), one deduces (17.1) for continuous convex functions from these inequalities.

The following result establishes the Schur inequalities for operators in a $I I_{1}$ factor.

Theorem 7.2. Let $\mathcal{A}$ be a MASA in $\mathcal{M}$ and let $E: \mathcal{M} \rightarrow \mathcal{A}$ be the tracepreserving conditional expectation. For every self-adjoint operator $A$ in $\mathcal{M}$, the spectral distribution of $B=E(A)$ is related to that of $A$ by $m_{B} \preceq m_{A}$.

Proof. Let $[a, b]$ be the smallest closed interval containing $\sigma(A) \cup \sigma(B)$. Since both $m_{B}$ and $m_{A}$ are probability measures, Proposition 3.1 implies that $m_{B} \preceq m_{A}$ iff for every continuous convex function $f \in C[a, b]$,

$$
\int_{I} f(\lambda) d m_{B}(\lambda) \leq \int_{I} f(\lambda) d m_{A}(\lambda) .
$$

Since the left side is $\tau(f(B))=\tau(f(E(A)))$ and the right side is $\tau(f(A))$, the preceding inequality follows from formula (7.1).

Theorem 7.2 makes the following assertion about the norm-closed unitary orbit $\mathcal{O}_{A}$ of a self-adjoint operator: $E\left(\mathcal{O}_{A}\right)$ is contained in the set of all selfadjoint operators $B \in \mathcal{A}$ satisfying $m_{B} \preceq m_{A}$. Thus, an affirmative reply to the following question would appear to be a natural counterpart of Horn's Theorem for $n \times n$ matrices.

Problem. Let $\mathcal{A}$ be a MASA in a $I I_{1}$ factor $\mathcal{M}$, let $E: \mathcal{M} \rightarrow \mathcal{A}$ be the trace-preserving conditional expectation, and let $A$ be a self-adjoint operator in $\mathcal{M}$. Does $E\left(\mathcal{O}_{A}\right)$ contain the set of all self-adjoint operators $B \in \mathcal{A}$ satisfying $m_{B} \preceq m_{A}$ ?

\section{REFERENCES}

[Apo57] T. Apostol. Mathematical Analysis. Addison-Wesley, Reading, MA, 1957.

[Ati82] M. Atiyah. Convexity and commuting Hamiltonians. Bull. L.M.S., 14(1):1-15, 1982.

[Bha87] R. Bhatia. Perturbation bounds for matrix eigenvalues. Pitman Research Notes in Mathematics. Longman, New York, 1987. 
[Fri00] S. Friedland. Finite and infinite dimensional generalizations of Klyachko's theorem. Lin. Alg. App., 319:3-22, 2000.

[GS82] V. Guillemin and S. Sternberg. Convexity properties of the moment mapping. Invent. Math., 67(3):491-513, 1982.

[Har34] Hardy, G.H., Littlewood J.E. and Polya, G. Inequalities. Cambridge University Press, Cambridge, 1934.

[Hor54] A. Horn. Doubly stochastic matrices and the diagonal of a rotation matrix. Amer. J. Math., 76(3):620-630, July 1954.

[Hor62] A. Horn. Eigenvalues of sums of hermitian matrices. Pac. J. Math., 12:225-241, 1962.

[Kad02a] R. Kadison. The Pythagorean theorem I: the finite case. Proc. N.A.S. (USA), 99(7):4178-4184, 2002.

[Kad02b] R. Kadison. The Pythagorean theorem II: the infinite discrete case. Proc. N.A.S. (USA), 99(8):5217-5222, 2002.

[Kad04] Richard V. Kadison. Non-commutative Conditional expectations and their applications, volume 365 of Contemporary Mathematics, pages 143-179. Amer. Math. Soc., 2004. Operator algebras, quantization, and noncommutative geometry.

[Kly98] A. Klyachko. Stable bundles, representation theory, and hermitian operators. Selecta Math, 4:419-445, 1998.

[Knu00] A. Knutson. The symplectic and algebraic geometry of Horn's problem. Lin. Alg. Appl., 319:61-81, 2000.

[KT99] A. Knutson and T. Tao. The honeycomb model of $G L(n)$ tensor products I: proof of the saturation conjecture. Jour. A.M.S., 12(4):1055-1090, 1999.

[Neu99] A. Neumann. An infinite-dimensional generalization of the Schur-Horn convexity theorem. Jour. Funct. Anal., 161(2):418-451, 1999.

[Neu02] A. Neumann. An infinite-dimensional generalization of the Kostant convexity theorem. Jour. Funct. Anal., 189(1):80-131, 2002.

[Pow67] R.T. Powers. Representations of uniformly hyperfinite algebras and their associated von Neumann rings. Ann. Math., 186:138-171, 1967.

[Sch23] I. Schur. Über eine klasse von mittlebildungen mit anwendungen auf der determinantentheorie. Sitzungsber. Berliner Mat. Ges., 22:9-29, 1923.

[She05] D. Sherman. Unitary orbits of normal operators in von Neumann algebras. preprint, 2005.

[Wey12] H. Weyl. Das asymptotische verieilungsgesetz der eigenwerte linearer partieller differentialgleichungen. Math. Ann., 71:441-479, 1912.

[Wey49] H. Weyl. Inequalities between the two kinds of eigenvalues of a linear transformation. Proc. N.A.S. (USA), 35:408-411, 1949.

Department of Mathematics, University of California, Berkeley, CA 94720, and Department of Mathematics, University of Pennsylvania, Philadelphia, PA 19104

E-mail address: arveson@mail.math.berkeley.edu, kadison@math.upenn.edu 